\title{
International Market Development
}

$\mathrm{I}_{\mathrm{si}}^{\mathrm{n}}$ ausländische Märkte einzutreten und sie gezielt zu bearbeiten, ist für viele Unternehmen heute selbstverständlich. Führende schweizerische und deutsche Unternehmen wie ABB, BASF und die UBS erzielen den größten Teil ihres Umsatzes im Ausland. Aber nicht nur Großunternehmen, sondern auch kleine und mittlere Unternehmen zeigen ein starkes internationales Engagement. Einerseits liegen die Potenziale für Spezialitäten in der ganzen Welt, andererseits lässt sich damit die Abhängigkeit von den Heimmärkten reduzieren.

Die turbulenten wirtschaftlichen Entwicklungen der letzten Monate haben drastisch aufgezeigt, wie dicht und unüberschaubar internationale Märkte zusammenhängen. In der gegenwärtigen Wirtschaftssituation geht es in vielen Unternehmen eher um Schadensbegrenzung als um den Ausbau strategischer Wachstumspotenziale. Gerade deshalb ist es wichtig, die Organisation der Marktpräsenz, die Form der Bearbeitung und die Steuerung der lokalen Aktivitäten genau abzustimmen. Auch gilt es abzuschätzen, welche Kosten bei Umsatzeinbrüchen sofort gesenkt werden und wo es durchzuhalten gilt. Dabei ist wichtig, wann ein neuer Aufschwung erwartet wird.

„International Market Development“ entwickelt und koordiniert internationale Märkte. Herausforderungen sind beispielsweise Marktprioritäten, Umgang mit kulturellen und sprachlichen Unterschieden, Zielkonflikten und Schnittstellen. Damit berücksichtigen wir nicht nur den Eintritt in ausländische Märkte, sondern auch die erfolgreiche Koordination zwischen globalem und lokalem Vorgehen, zwischen bestehenden und neuen Märkten. Drei Fragen stehen im Vordergrund und prägen den Aufbau des Heftes:

- strategische und organisatorische Dilemmas,

- operative Instrumente und Prozesse,

- Kontrolle und Marktforschung.

Die Ausgabe zeigt auf, mit welchen Herausforderungen Unternehmen heute bei der Erschließung neuer Märkte konfrontiert wer- den und welche Lösungen eingesetzt werden, um diese zu bewältigen. Dabei stehen die Prozesssicht und die Besonderheit einzelner Märkte im Vordergrund. Welche Schritte über die Vorselektion und Auswahl der Märkte bis hin zum Markteintritt sind von Unternehmen zu wählen? Welche Organisationsform ist für eine Marktpräsenz geeignet? Wie unterscheidet sich zum Beispiel das erfolgreiche Vorgehen in Indien von den Schritten, die in Zentral- oder Osteuropa den Markt eröffnen?

Das Thema ist von hoher praktischer Relevanz, sie wird durch die aktuelle Wirtschaftslage akzentuiert. Auch in der internationalen Forschungsgemeinschaft sind die Themen der internationalen Markterschließung und -entwicklung verankert.

Hier setzen die Beiträge der aktuellen Ausgabe der Marketing Review St. Gallen an. In bewährter Form setzen sich Autoren aus Praxis und Forschung mit spezifischen Herausforderungen des International Market Development auseinander. Wir danken den beteiligten Autoren, Gutachtern und Koordinatoren für ihre aufwändige Mitwirkung und freuen uns, dem Leser ein Gesamtbild mit konkreten Impulsen zum Thema präsentieren zu können. Rückmeldungen zu dieser Ausgabe sind stets willkommen!
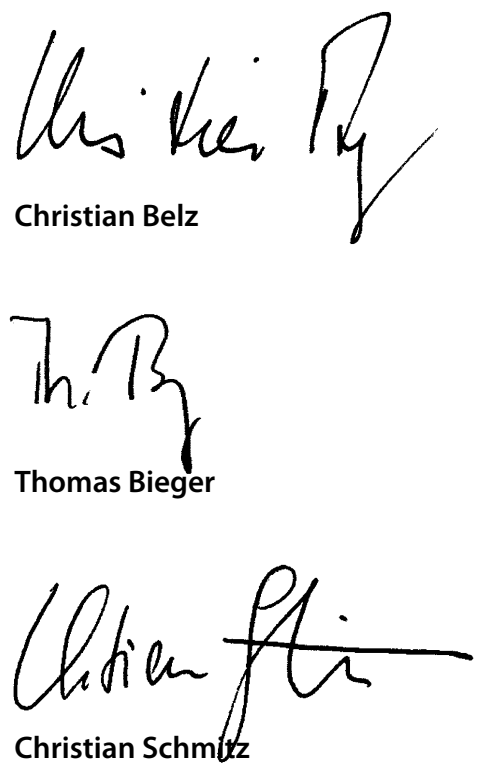

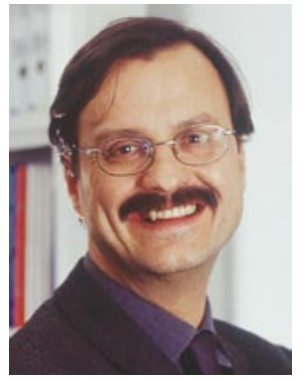

Christian Belz

Prof. Dr. Christian Belz, Ordinarius für Betriebswirtschaftslehre mit besonderer Berücksichtigung des Marketing an der Universität St. Gallen und Geschäftsführender Direktor des dortigen Instituts für Marketing sowie Mitherausgeber der Marketing Review St. Gallen

E-Mail: christian.belz@unisg.ch

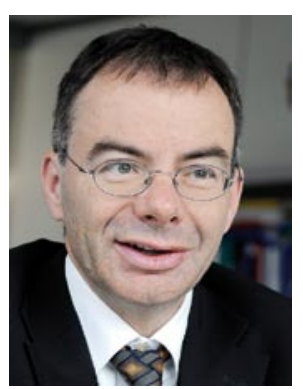

Thomas Bieger

Prof. Dr. Thomas Bieger, Ordinarius für Betriebswirtschaftslehre mit besonderer Berücksichtigung der Tourismuswirtschaft und Leiter des Instituts für öffentliche Dienstleistungen und Tourismus an der Universität St. Gallen sowie Mitherausgeber der Marketing Review St. Gallen E-Mail: thomas.bieger@unisg.ch

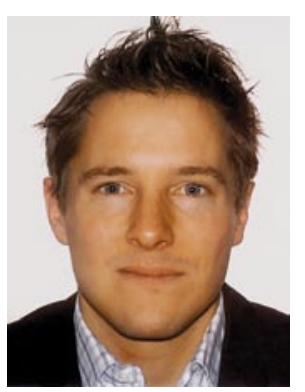

Christian Schmitz

Dr. Christian Schmitz, Habilitand, Lehrbeauftragter für Marketing und Leiter des Kompetenzzentrums für Business-to-Business Marketing am Institut für Marketing, Universität St. Gallen E-Mail:christian.schmitz@unisg.ch 